\title{
On the Probabilistic Structure of Quasi-free States of a Clifford Algebra
}

\author{
M. Sirugue-Collin \\ F-13274 Marseille Cedex 2, France

\section{Sirugue} \\ Centre de Physique Théorique, CNRS, F-13274 Marseille Cedex 2, France
}

Université de Provence, U.E.R de Physique, Marseille, and Centre de Physique Théorique, CNRS,

\begin{abstract}
We prove that the correlation functions of a non-relativistic Fermi field given by a quasi-free state are directly related to the values of the characteristic function of a probability measure over the phase space of a classical spin system.
\end{abstract}

\section{Introduction}

In the past few years probabilistic methods have been proved to be very useful in constructive field theory and especially for the study of Schwinger functions. Indeed the Schwinger functions of a Bose field are symmetric functions of their arguments, hence they can be the moments of a probability measure; moreover for the free Bose field the Wick theorem leads to a gaussian measure [1].

However for fermions and except for some results [2,3], the situation is less clear, even for the free Fermi field, since the Schwinger functions are completly antisymmetric with respect to their arguments. Consequently it is a priori impossible to repeat for fermions what has been done for bosons.

Our aim with this note is to present an attempt to bypass this difficulty. Namely what we prove is that, given a quasi-free state over a Clifford algebra, the correlation functions are, up to a trivial factor, the values of the characteristic function of a probability measure over the phase space of a classical spin system. [Theorem (2.26).]

Moreover we show that this theorem is the analogue of what can be done for boson systems.

\section{Quasi-free States as Given by the Characteristic Function of a Probability Measure}

To fix the notations we repeat some definitions and results which can be found in an extended form in [4]. 
$\mathfrak{H}$ is a real separable Hilbert space of even or infinite dimension with a symmetric, real-valued, positive-definite, scalar product $S$. The Clifford algebra $\overline{\mathfrak{A}(\mathfrak{H}, S)}$ is the $C^{*}$-algebra generated by the $b(\varphi), \varphi \in \mathfrak{H}$, which satisfy

(2.1) $b(\alpha \varphi+\beta \psi)=\alpha b(\varphi)+\beta b(\psi), \quad \forall \alpha, \beta \in \mathbb{R}, \varphi, \psi \in \mathfrak{H}$,

(2.2) $b(\varphi)^{*}=b(\varphi), \quad \forall \varphi \in \mathfrak{H}$,

(2.3) $\quad b(\varphi)^{2}=S(\varphi, \varphi) \mathbb{1}, \quad \forall \varphi \in \mathfrak{H}$.

A quasi-free state over $\overline{\mathfrak{A}(\mathfrak{H}, S)}$ is a state of $\overline{\mathfrak{A}(\mathfrak{H}, S)}$ which satisfies

(2.4) $\omega(b(\varphi))=0, \quad \forall \varphi \in \mathfrak{H}$,

(2.5) $\omega\left(\dot{b}\left(\varphi_{1}\right) b\left(\varphi_{2}\right) \ldots b\left(\varphi_{n}\right)\right)$

$$
=\sum_{i=2}^{n}(-1)^{i} \omega\left(b\left(\varphi_{1}\right) b\left(\varphi_{i}\right)\right) \omega\left(b\left(\varphi_{2}\right) \ldots b\left(\varphi_{i}\right) \ldots b\left(\varphi_{n}\right)\right),
$$

$\forall \varphi_{i} \in \mathfrak{H}$; namely it satisfies the Wick theorem. As a consequence it is completely defined by its two-point function:

$$
\omega(b(\varphi) b(\psi))=S(\varphi, \psi)+i S(A \varphi, \psi), \quad \forall \varphi, \psi \in \mathfrak{H} .
$$

where $A$ is a (real) linear operator on $\mathfrak{H}$ such that

(2.7) $S(A \varphi, \psi)=-S(\varphi, A \psi), \quad \forall \varphi, \psi \in \mathfrak{H}$,

(2.8) $\|A\| \leqq 1$.

Vice-versa, given a real linear operator $A$ on $\mathfrak{H}$ which satisfies (2.7) and (2.8), then there exists a quasi-free state $\omega_{A}$ of $\overline{\mathfrak{A}(\mathfrak{H}, S)}$ whose two-point function is given by (2.6).

Let $A=|A| J$ be the polar decomposition of $A$ which satisfies (2.7) and (2.8), then $J$ satisfies

$$
J^{2}=-\mathbb{1}
$$

$$
S(J \varphi, \psi)=-S(\varphi, J \psi), \quad \forall \varphi, \psi \in \mathfrak{H} .
$$

In what follows we shall restrict ourselves to those $A$ 's for which $|A|$ has a pure point spectrum. This is not a too severe restriction since in each quasi-equivalence class of $\omega_{A}$ there exists a quasi-free state $\omega_{A^{\prime}}$ for which $\left|A^{\prime}\right|$ has a pure point spectrum (see e.g. [5-7]).

Consequently there exists an orthonormal basis $\left\{e_{i}, f_{i}\right\}_{i=1,2, \ldots}$ of $\mathfrak{S}$ such that

(2.10) $J e_{i}=f_{i}, \quad J f_{i}=-e_{i}$,

(2.11) $|A| e_{i}=\operatorname{th}\left(\theta_{i}\right) e_{i}$,

(2.12) $|A| f_{i}=\operatorname{th}\left(\theta_{i}\right) f_{i}$,

(2.13) $\theta_{i} \in \mathbb{R}^{+}$or $+\infty$.

We shall restrict ourselves to $\theta_{i}<\infty$, which is possible if we consider the $\omega_{A}$ up to quasi-equivalence. 
Moreover using the result of [8] $\omega_{A}$ is a product state, namely

$$
\begin{aligned}
& \omega_{A}\left(b\left(x_{i_{1}}\right) b\left(y_{i_{1}}\right) b\left(x_{i_{2}}\right) b\left(y_{i_{2}}\right) \ldots b\left(x_{i_{p}}\right) b\left(y_{i_{p}}\right)\right) \\
& \quad=\omega_{A}\left(b\left(x_{i_{1}}\right) b\left(y_{i_{1}}\right)\right) \omega_{A}\left(b\left(x_{i_{2}}\right) b\left(y_{i_{2}}\right)\right) \ldots \omega_{A}\left(b\left(x_{i_{p}}\right) b\left(y_{i_{p}}\right)\right),
\end{aligned}
$$

where $x_{i_{j}}, y_{i_{j}}$ belong to the space generated by $\left(e_{i_{j}}, f_{i_{j}}\right)$ and all $i_{j}$ are distinct.

Now we shall give another description of the Clifford algebra which is convenient for what follows in the sense that it bears a strong similarity to the algebra of commutation relations $[9,10]$. We shall not be systematic in order to make clear the correspondance between the two descriptions.

Let $2 N$ (resp. $\infty$ ) be the real dimension of $\mathfrak{H}$ and $G$ the group of subsets of a set with $N$ elements (resp. the set of finite subsets of the set of natural integers) endowed with the symmetric difference as product; this group has been already considered by Ginibre in [11]. If $g \in G \times G$ :

$$
g=\left(\left(i_{1}, \ldots, i_{p}\right),\left(j_{1}, \ldots, j_{q}\right)\right)
$$

one defines (one can assume $i_{1}<i_{2} \ldots<i_{p}, j_{1}<j_{2} \ldots<j_{q}$ ):

$$
\delta_{g}=i^{(p+q)(p+q-1) / 2} b\left(e_{i_{1}}\right) \ldots b\left(e_{i_{p}}\right) b\left(f_{j 1}\right) \ldots b\left(f_{\jmath q}\right) .
$$

The factor $i^{(p+q)(p+q-1) / 2}$ is such that $\delta_{g}$ is self-adjoint. Then it is not difficult to realize that:

$$
\delta_{g} \delta_{g^{\prime}}=\xi\left(g, g^{\prime}\right) \delta_{g * g^{\prime}}, \quad \forall g, g^{\prime} \in G \times G,
$$

where $\varrho * \varrho^{\prime}$ stands for the product within $G \times G$ and $\xi$ is an exponent of $G \times G$, i.e. defines a central extension of $G \times G$.

Let us specialize to the case where the real dimension of $\mathfrak{H}$ is two. Let $(e, f)$ be the orthonormal basis which diagonalizes $|A|$. $G \times G$ is the group of subsets of a set with two elements $\{1,2\}$ and isomorphic to $S_{2} \times S_{2}\left(S_{2}\right.$ is the group of two elements)

$$
G \times G=\{\emptyset,\{1\},\{2\},\{1,2\}\},
$$

then

$$
\delta_{0}=\mathbb{1}, \quad \delta_{\{1\}}=b(e), \quad \delta_{\{2\}}=b(f), \quad \delta_{\{1,2\}}=i b(e) b(f) .
$$

From which the Clifford algebra generated by $b(e), b(f)$ appears as the group algebra of the central extension of $S_{2} \times S_{2}$ by an exponent $\xi$ which one can easily calculate from (2.19).

Let us consider on this Clifford algebra [resp. on $\overline{\Delta(G \times G, \xi)}]$ the state $\omega_{A}$ (resp. the state $\varrho_{A}$ ) such that:

$$
\begin{aligned}
\varrho_{A}\left(\delta_{\vartheta}\right) & =\omega_{A}(\mathbb{1})=1, \\
\varrho_{A}\left(\delta_{\{1\}}\right) & =\varrho_{A}\left(\delta_{\{2\}}\right)=\omega_{A}(b(e))=\omega_{A}(b(f))=0, \\
\varrho_{A}\left(\delta_{\{1,2\}}\right) & =i \omega_{A}(b(e) b(f))=-\operatorname{th}(\theta), \quad \theta \in \mathbb{R}^{+} .
\end{aligned}
$$

One can formulate the following lemma:

Lemma (2.23). $\varrho_{A}$ as a function of the group $S_{2} \times S_{2}$ is the characteristic function of a probability measure on the dual group $\hat{S}_{2} \times \hat{S}_{2}$. 
Indeed $\hat{S}_{2}$ has two elements $\sigma_{1}$ and $\sigma_{2}\left(\sigma_{1}\right.$ is the trivial character $)$ so that $\hat{S}_{2} \times \hat{S}_{2}=\left\{\left(\sigma_{1}, \bar{\sigma}_{1}\right),\left(\sigma_{1}, \bar{\sigma}_{2}\right),\left(\sigma_{2}, \bar{\sigma}_{1}\right),\left(\sigma_{2}, \bar{\sigma}_{2}\right)\right\}$; one can write

$$
\varrho_{A}\left(\delta_{g}\right)=\sum_{\sigma_{l}, \bar{\sigma}_{J}} \mu_{A}\left(\sigma_{i}, \bar{\sigma}_{j}\right)\left(\sigma_{i}, \bar{\sigma}_{j}\right)(g), \quad \forall g \in S_{2} \times S_{2}
$$

and $\left(\sigma_{i}, \bar{\sigma}_{j}\right)(g)$ is the value of the character $\left(\sigma_{i}, \bar{\sigma}_{j}\right) \in \hat{S}_{2} \times \hat{S}_{2}$ on $g$.

An easy computation shows that

$$
\begin{array}{ll}
\mu_{A}\left(\sigma_{i}, \bar{\sigma}_{j}\right)=e^{-\theta} / 4 \operatorname{ch}(\theta) & \text { if } \quad i=j, \\
\mu_{A}\left(\sigma_{i}, \bar{\sigma}_{j}\right)=e^{+\theta} / 4 \operatorname{ch}(\theta) & \text { if } \quad i \neq j ;
\end{array}
$$

this measure is of Gibbsian form, namely $\mu_{A}=Z^{-1} e^{-H}$, and $H$ is of ferromagnetic type (see e.g. [11]).

If we gather this result with formula (2.14) we have the following theorem.

Theorem (2.26). Let $\omega_{A}$ be a quasi-free state of $\overline{\mathfrak{U}(\mathfrak{H}, S)}(\operatorname{dim} \mathfrak{H}=\infty)$ such that if $A=|A| J$ is the polar decomposition of $A$ then $|A|$ has a purely discrete spectrum.

Let $\left(e_{i}, f_{i}\right)_{i=1,2, \ldots, n, \ldots}$ be the orthonormal basis of $\mathfrak{H}$ which diagonalizes $|A|$ and such that $J e_{i}=f_{i}$; let $G$ be the group of finite subsets of the set of natural integers equipped with the symmetric difference as product; then there exists a probability measure $\mu_{A}$ on $\hat{G} \times \hat{G}$ such that

$$
\begin{aligned}
& \omega_{A}\left(b\left(e_{i_{1}}\right) b\left(e_{i_{2}}\right) \ldots b\left(e_{i_{p}}\right) b\left(f_{j_{1}}\right) \ldots b\left(f_{j_{q}}\right)\right) \\
& \quad=(-i)^{(p+q)(p+q-1) / 2}(-1)^{\delta} \int_{\hat{G} \times \hat{G}} d \mu_{A}(\sigma, \bar{\sigma})(\sigma, \bar{\sigma})\left(\left(i_{1} \ldots i_{p}\right),\left(j_{1} \ldots \dot{j}_{q}\right)\right)
\end{aligned}
$$

where one has assumed that $i_{1}<i_{2} \ldots<i_{p}, j_{1}<\ldots<j_{q} .(-1)^{\delta}$ is the parity of the permutation which brings $i_{1} \ldots i_{p} j_{1} \ldots j_{q}$ to $k_{1}, \ldots k_{p+q}\left(k_{r}=i_{r}\right.$ or $\left.j_{r}\right)$ and $k_{1} \leqq k_{2}, \ldots \leqq k_{p+q}$. $(\sigma, \bar{\sigma})\left(\left(i_{1}, \ldots, i_{p}\right),\left(j_{1} \ldots j_{q}\right)\right)$ denotes the value of the character $(\sigma, \bar{\sigma})$ of $\hat{G} \times \hat{G}$ on an element $\left(\left(i_{1} \ldots i_{p}\right),\left(j_{1}, \ldots, j_{q}\right)\right.$ of $G \times G$.

There is an analogue of this formula for bosons; indeed let us consider a onedimensional harmonic oscillator with canonical (quantum) variables $p$ and $q$; let $\Omega$ be its ground state; then an obvious calculation shows that:

$$
\left(\Omega \mid e^{i(\lambda p+\mu q)} \Omega\right)=\int_{-\infty}^{+\infty} \int_{-\infty}^{+\infty} d \mu_{\Omega}(\hat{u}, \hat{v}) e^{-i(\lambda \hat{u}+\mu \hat{v})},
$$

where

$$
d \mu_{\Omega}(\hat{u}, \hat{v})=\left[\iint d \hat{u} d \hat{v} \exp (-(2 / \hbar \omega) \mathscr{H}(\hat{u}, \hat{v}))\right]^{-1} e^{-(2 / \hbar \omega) \mathscr{H}(\hat{u}, \hat{v})} d \hat{u} d \hat{v},
$$

and $\mathscr{H}(\hat{u}, \hat{v})$ is the classical Hamiltonian.

Let us make some further remarks: another proof of the theorem would be to use the fact that $\omega_{A}$ is non zero on a maximal abelian subalgebra of $\overline{\mathfrak{A}(\mathfrak{H}, S)}$; namely the one generated by the $i b\left(e_{i}\right) b\left(f_{i}\right)$; hence its restriction to this abelian subalgebra is the Fourier transform of a probability measure over the space of its characters. The proof can be completed by using Theorem (2.14) in [12]. This shows that the situation we have here is more general than the one in formula (2.27) where the essential point is that a Gaussian is a positive type function on $\mathbb{R}^{2}$ and on the Weyl group.

On the other hand it is clear that $\hat{G}$ is isomorphic to a classical spin system (see [11]); in this sense we can say that the classical spin system is the analogue 
of the classical phase space for the Fermi system we have considered. Moreover the measure of Theorem (2.26) is of Gibbsian form for a ferromagnetic interaction between the classical spins.

\section{References}

1. See e.g. Guerra,F.: In: Constructive quantum field theory (ed. G. Velo, A. Wightman). BerlinHeidelberg-New York: Springer 1973

2. Berezin, F.A.: The method of second quantization. New York: Academic Press 1966

3. Gross, L.: J. Funct. Anal. 10, 52 (1972)

Frohlich, J., Osterwalder, K.: Helv. Phys. Acta 47, 781 (1974)

4. Manuceau, J., Rocca, F., Sirugue, M., Verbeure, A.: In: Cargèse lectures in physics (ed. D. Kastler). New York: Gordon and Breach 1970

5. Rideau, G.: Commun. math. Phys. 9, 229 (1968)

6. Powers, R., Stormer, E.: Commun. math. Phys. 16, 1 (1970)

7. Manuceau, J., Verbeure, A.: Ann. Inst. Henri Poincaré 16, 87 (1971)

8. Manuceau, J., Rocca,F., Testard, D.: Commun. math. Phys. 12, 43 (1969)

9. Segal,I.E.: Math. Scand. 13, 31 (1963)

10. Slawny, J.: On factor representations and the $C^{*}$-algebra of C.C.R. Preprint 1971

11. Ginibre, J.: In: Cargèse lectures in physics (ed. D. Kastler). New York: Gordon and Breach 1970

12. Beaume, R., Manuceau, J., Pellet, A., Sirugue, M.: Commun. math. Phys. 38, 29 (1974)

Communicated by H. Araki

Received September 19, 1975; in revised form January 21, 1976 
